\title{
Eficacia de dos fitasas bacterianas en la liberación de fósforo en dietas para pollos de engorda en crecimiento
}

\author{
Efficacy of two bacterial phytase in the release of phosphorus in \\ diets for growing chicken broilers
}

\author{
María Liliana Diosdado Espinozaa , Arturo Cortes Cuevasa ${ }^{a}$ Ernesto Avila González ${ }^{a}$
}

\begin{abstract}
RESUMEN
La eficacia de dos fitasas (Citrobacter brakii y $E$. Coli) utilizadas en diferentes dosis (500 y 1,000 FTU) se evaluaron en una dieta deficiente en fósforo ( $0.15 \%$ P disponible) formulada a base de sorgo-soya. Para la estimación de la eficacia en la liberación de $\mathbf{P}$ de las fitasas evaluadas se utilizó como referencia fósforo inorgánico suplementado a través de fosfato monodicálcico (FMD), para alcanzar una concentración final de $\mathbf{P}$ disponible de $0.23,0.31$ y $0.39 \%$. Para lo anterior la dieta basal deficiente en $\mathbf{P}$ se suplementó como sigue: 1) sin fitasas, sin FMD; 2) 0.08\% FMD; 3) 0.16\% FMD; 4) 0.24\% PMD; 5) citrobacter-500 FTU; 6) coli-500 FTU; 7) citrobacter-1,000 FTU y 8) coli-1,000 FTU. Los tratamientos se aplicaron a 216 pollos de la estirpe Ross (27 pollos/ tratamiento) de los 7 a 21 días de edad. La ganancia de peso, eficiencia alimenticia y concentración de ceniza y P en tibias fue mayor en los pollos que recibieron 0.39\% PMD y los 1,000 FTU de ambas fitasas, no existiendo diferencias en estos niveles entre los tratamientos FMD y fitasas. La cantidad de fósforo liberado no se afectó ( $P>0.05$ ) por el tipo de fitasa (promedio $0.142 \%$ ), pero sí por el nivel de fitasa utilizado (0.102 vs $0.182 \%$ para 500 y 1,000 FTU respectivamente). Los resultados indican que la suplementación con 1,000 FTU/ kg de ambas fitasas en dietas deficientes en P resulta en ganancias y eficiencias alimenticias similares cuando las dietas se suplementan hasta $0.24 \%$ de FMD. Ambas fitasas mejoraron en $78.4 \%$ el aprovechamiento del fósforo fítico en dietas sorgo-soya para pollos en crecimiento.
\end{abstract}

PALABRAS CLAVE: Fitasas, Citrobacter braakii, Escherichia coli, Fósforo en tibias, Cenizas en tibias, Pollos de engorda.

\begin{abstract}
The efficacy of two phytases (Citrobacter brakii and Escherichia coll) used in different doses (500 and 1,000 FTU) were evaluated in a diet deficient in phosphorus $(0.15 \%$ available $P)$ formulated with sorghum-soybean meal. To estimate the efficacy of the release of $\mathbf{P}$ evaluated phytases, inorganic phosphorus supplemented by mono-phosphate (MDP) to reach a final concentration of available $P$ of $0.23,0.31$ and $0.39 \%$ was used as reference. The basal diet deficient in $P$ was supplemented as follows: 1 ) Without phytases, without MDP; 2) $0.08 \%$ MDP; 3) $0.16 \%$ MDP; 4) $0.24 \%$ MDP; 5) Citrobacter-500 FTU; 6) coli FTU-500; 7) citrobacter1,000 FTU and 8) FTU coli-1,000. The treatments were applied to Ross 216 chickens ( 27 birds/ treatment) 7 to $21 \mathrm{~d}$ old. Weight gain, feed efficiency and concentration of tibia ash and P was better in chickens receiving $0.39 \%$ PMD and 1,000 FTU of both phytase, with no differences in these levels between MDP and phytase treatments. The amount of phosphorus released was not affected ( $P>0.05$ ) by type of phytase (average $0.142 \%$ ), with effect to level of phytase used $(0.102$ vs $0.182 \%$ for 500 and 1,000 FTU respectively). The results indicate that supplementation 1,000 FTU / $\mathrm{kg}$ in both phytase in diets deficient in P results in weight gain and feed efficiency similar when the diets are supplemented with $0.24 \%$ MDP. Both phytases improved to $78.4 \%$ utilization of phytate phosphorus in sorghum-soybean meal diets on growing chicks.
\end{abstract}

KEY WORDS: Phytases, Citrobacter braakii, Escherichia coli, Phosphorus tibia, Tibia ashes, Broilers.

Recibido el 15 de marzo de 2016. Aceptado el 17 de agosto de 2016.

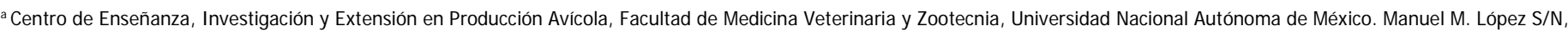
Col. Zapotitlán, 13209 Tláhuac DF. Tel: 584515 30. México.

Autor de correspondencia: cortescuevasarturo@yahoo.com. 


\section{INTRODUCCIÓN}

El fósforo es un elemento importante para sostener la producción de alimentos de origen animal, las reservas mundiales de fosfatos son limitadas y en un futuro será un problema para obtener las cantidades suficientes para proveer de este compuesto a la industria de los alimentos balanceados para animales, lo que resulta en un desafío poder sostener dicha demanda ${ }^{(1,2)}$.

Para el sector avícola esto implica que la utilización de fósforo por los animales deberá ser explorada para un máximo aprovechamiento de este mineral en las dietas. Esto incluye más estudios sobre el uso de aditivos, especialmente las fitasas como una herramienta en la eficiencia para extraer más fósforo en las dietas vegetales, ya que del 50 al $85 \%$ del fósforo almacenado en los granos está ligado al ácido fítico y sus sales ${ }^{(3,4)}$.

La utilización de las fitasas en la industria de alimentos balanceados se ha visto favorecida por lo atractivo de sus precios, la mejora en la utilización del fósforo fítico, en el aumento del rendimiento animal (carne y hueso) y la disminución del grado de daño ambiental (menor eliminación de fósforo al ambiente a través de las excretas) ${ }^{(5)}$.

Las fitasas liberan fósforo a partir de fósforo fítico, y como resultado de la destrucción de este factor anti-nutricional, permiten reducir el uso de fuentes de fósforo inorgánico en la dieta(6). Como resultado, el uso de fitasas mejora la digestibilidad no sólo del fósforo y cationes divalentes como $\mathrm{Ca}$, Mg y Zn, sino también de energía y proteína(7).

En la actualidad, las fitasas recientes de origen bacteriano brindan una mayor estabilidad en $\mathrm{pH}$ bajos, y soportan temperaturas elevadas a los que son sometidos los alimentos balanceados para aves sin perder sus propiedades. Dentro de las bacterias utilizadas para la obtención de fitasas, destacan aquéllas provenientes de Escherichia coli y Citrobacter braakif ${ }^{8,9}$. Cada fitasa ofrece beneficios en su uso e incluso existen diferentes presentaciones del producto comercial.

Una dosis equivalente a $500 \mathrm{FTU} / \mathrm{kg}$ de $E$. coli libera de 20 a $30 \%$ más nutrientes que la misma dosis de una fitasa de Aspergillus spp, y su efecto es más consistente debido a su pH y una mayor estabilidad durante la digestión por pepsina en el tracto intestinal ${ }^{(10)}$.

Utilizar una alta concentración de fitasa (megadosis) en las dietas, en busca de una rápida reducción de la concentración de fitato en el estómago o proventrículo, reduce así el efecto antinutricional del fitato, e incrementa aún más la liberación de fósforo(11). De esta manera se logra la máxima destrucción del fitato, de tal forma que el tipo de fitasas, niveles de dosificación y el contenido de fitato de la dieta van a tener un papel importante en la respuesta productiva(12). Estudios recientes indican que niveles elevados de fitasa (megadosis) en la dieta de los pollos (1,000 y 2,000 FTU/ $\mathrm{kg}$ ) mejoran la liberación de fósforo $(0.15 \%)$ y calcio $(0.18 \%)$ e incrementan los parámetros productivos $^{(9)}$.

Con estos antecedentes, se planteó el presente estudio para conocer la liberación de fósforo en relación a fosfato monodicálcico en dietas sorgo+soya para pollos en crecimiento deficientes en fósforo disponible, mediante la suplementación de dos fuentes de fitasas (Citrobacter braakii y Escherichia col/) a diferentes dosis (500 y megadosis de $1,000 \mathrm{FTU} / \mathrm{kg}$ ).

\section{MATERIAL Y MÉTODOS}

El experimento se realizó en una caseta de ambiente natural, localizada en el Centro de Enseñanza, Investigación y Extensión en Producción Avícola de la Universidad Nacional Autónoma de México. Se utilizaron 216 pollos machos de la estirpe Ross 308 de siete días de edad, distribuidos en ocho tratamientos con tres repeticiones de nueve aves cada uno (27 animales por tratamiento). Los pollos se alojaron en pisos de jaulas en baterías tipo Petersime, con calefacción eléctrica y temperatura regulada con termostato. El alimento y el agua de bebida se ofrecieron a libre acceso. Los pollos de 1 a 6 días de edad, se alimentaron con dietas sorgosoya, formulada para cubrir todas las recomendaciones nutricionales de la estirpe. A los siete días de edad se distribuyeron en forma completamente al azar en ocho tratamientos: 
1) Dieta basal sorgo-soya, deficiente en fósforo disponible $(0.15 \%)$.

2) Como $1+0.08 \%$ de fósforo inorgánico a partir de fósforo monodicálcico (FMD).

3) Como $1+0.16 \%$ de fósforo inorgánico a partir de FMD.

4) Como $1+0.24 \%$ de fósforo inorgánico a partir de FMD.

5) Como $1+500$ FTU* de fitasa microbiana de Citrobacter braakii.

6) Como $1+500$ FTU de fitasa microbiana de Escherichia coli.

7) Como $1+1,000$ FTU de fitasa microbiana de Citrobacter braakii.

8) Como $1+1,000$ FTU de fitasa microbiana de Escherichia coli.

*FTU = cantidad de enzima necesaria para liberar $1 \mu \mathrm{mol}$ de fósforo inorgánico por minuto en un $\mathrm{pH}$ de 5.5 a $37^{\circ} \mathrm{C}$.

Para la estimación de la eficacia en la liberación de $\mathrm{P}$ se utilizó como referencia fósforo inorgánico (Pi) suplementado a través de fosfato monodicálcico (FMD) para alcanzar una concentración final de $\mathrm{Pi}$ disponible de $0.23,0.31$ y $0.39 \%$ (tratamientos 2,3 y 4). La dieta basal cubre todos los requerimientos con excepción de Pi (Cuadro 1); las adiciones de FMD y fitasas fueron sustituyendo a la celulosa en la dieta basal para no alterar el balance nutricional de las diferentes dietas experimentales.

El consumo de alimento se registró por réplica (por cada nueve pollos) y la ganancia diaria de peso se registró individualmente en una báscula electrónica Marca Torrey Mod. L-EQ 5/10. A los 21 días de edad, se sacrificó el $60 \%$ de los pollos con el método que indica la norma NOM-033-ZOO-1995, por medio de la sujeción segura del animal y de inmediato la dislocación cervical en un solo movimiento para evitar dolor innecesario; se les extrajo la tibia izquierda para determinar el contenido porcentual de cenizas y fósforo (de acuerdo al AOAC, 2006) ${ }^{(13)}$ respectivamente.

Los datos de crecimiento, consumo y de eficiencia alimenticia se analizaron utilizando el rodete como unidad experimental. El contenido de fósforo y porcentaje de cenizas en tibias, se analizaron como un diseño completamente al azar (SPSS para Windows versión 17.0). La biodisponibilidad del fósforo se estimó con curvas estándar a través de regresiones lineales simples de primer grado $(\mathrm{Y}=\beta \mathrm{O}+\beta 1 \mathrm{x})$, utilizando un paquete computacional SSPS versión $17.0^{(14)}$, a partir del contenido porcentual de fósforo y cenizas en tibias. Para la curva estándar se utilizaron los tratamientos 1, 2, 3 y 4. Para la curva de Citrobacter braakii los tratamientos 1, 5 y 7 . Finalmente para la de Escherichia coli fueron los tratamientos 1, 6 y 8.

Cuadro 1. Composición de la dieta basal experimental para pollos de 7 a 21 días de edad

\begin{tabular}{|c|c|}
\hline Ingrediente & $\mathrm{Kg}$ \\
\hline Sorgo & 627.06 \\
\hline Pasta de soya & 327.13 \\
\hline Carbonato de calcio & 22.91 \\
\hline Aceite de soya & 6.65 \\
\hline L-Lisina $\mathrm{HCl}$ & 4.80 \\
\hline Sal & 3.80 \\
\hline DL-Metionina & 3.30 \\
\hline Celulosa & 1.40 \\
\hline Cloruro de colina $60 \%$ & 1.00 \\
\hline Premezcla de vitaminas* & 1.00 \\
\hline Premezcla de minerales** & 0.50 \\
\hline Bacitracina de zinc 10\% & 0.30 \\
\hline Antioxidante ${ }^{\star \star \star}$ & 0.15 \\
\hline TOTAL & 1000.00 \\
\hline \multicolumn{2}{|l|}{ Análisis calculado } \\
\hline Energía metabolizable, Kcal/kg & 2950 \\
\hline Proteína cruda, \% & 22.00 \\
\hline Fósforo disponible no fítico determinado, \% & 0.15 \\
\hline Calcio total, \% & 1.02 \\
\hline Metionina + cistina digestible, $\%$ & 0.92 \\
\hline Lisina digestible, \% & 1.39 \\
\hline Treonina digestible, \% & 0.82 \\
\hline Sodio, \% & 0.18 \\
\hline \multicolumn{2}{|c|}{$\begin{array}{l}\text { *Premezcla de vitaminas por kg: Vitamina A 12'000,000 UI; vitamina D3 } \\
\text { 2'500,000 Ul; vitamina E } 15,000 \text { Ul; vitamina K3 2,000 mg/kg; vitamina B1 2,250 } \\
\mathrm{mg} / \mathrm{kg} \text {; vitamina B2 7,500 mg/kg; vitamina B3 } 45,000 \mathrm{mg} / \mathrm{kg} \text {; vitamina B5 12,500 } \\
\mathrm{mg} / \mathrm{kg} \text {; vitamina B6 3,500 mg/kg; vitamina B12 } 20 \mathrm{mg} / \mathrm{kg} \text {; ácido fólico 1,500 } \\
\mathrm{mg} / \mathrm{kg} \text {; biotina } 125 \mathrm{mg} / \mathrm{kg} \text {. }\end{array}$} \\
\hline \multicolumn{2}{|c|}{$\begin{array}{l}\text { **Premezcla de minerales por } 0.5 \mathrm{~kg} \text { : yodo } 300 \mathrm{mg} / \mathrm{kg} \text {; selenio } 200 \mathrm{mg} / \mathrm{kg} \text {; } \\
\text { cobalto } 200 \mathrm{mg} / \mathrm{kg} \text {; hierro 50,000 mg/kg; cobre 12,000 mg/kg; zinc 50,000 } \\
\mathrm{mg} / \mathrm{kg} \text {; manganeso 110,000 mg/kg; }\end{array}$} \\
\hline ***BHT (1.2 \%), BHA (9.0\%), Etoxiquin (4.8\%). & \\
\hline
\end{tabular}


Cuadro 2. Resultados promedio de rendimiento productivo y porcentajes de cenizas y fósforo inorgánico en tibias de pollos de 7-21 días de edad

\begin{tabular}{ccccc}
\hline Tratamientos & $\begin{array}{c}\text { Ganancia de peso } \\
(\mathrm{g})\end{array}$ & $\begin{array}{c}\text { Eficiencia alimenticia } \\
(\mathrm{kg}: \mathrm{kg})\end{array}$ & $\begin{array}{c}\text { Cenizas en tibias } \\
(\%)\end{array}$ & $\begin{array}{c}\text { Fósforo en tibias } \\
(\%)\end{array}$ \\
\hline 1 & $586 \pm 11.0^{\mathrm{a}}$ & $0.69 \pm 0.001^{\mathrm{a}}$ & $42.78 \pm 0.52^{\mathrm{a}}$ & $7.07 \pm 0.10^{\mathrm{a}}$ \\
2 & $649 \pm 10.1^{\mathrm{ab}}$ & $0.71 \pm 0.001^{\mathrm{ab}}$ & $46.45 \pm 0.46^{\mathrm{b}}$ & $7.29 \pm 0.11^{\mathrm{b}}$ \\
3 & $713 \pm 9.9^{\mathrm{b}}$ & $0.73 \pm 0.001^{\mathrm{b}}$ & $48.62 \pm 0.49^{\mathrm{c}}$ & $7.95 \pm 0.09 \mathrm{c}$ \\
4 & $737 \pm 10.7^{\mathrm{c}}$ & $0.76 \pm 0.001^{\mathrm{c}}$ & $48.87 \pm 0.51^{\mathrm{d}}$ & $8.00 \pm 0.09^{\mathrm{d}}$ \\
5 & $673 \pm 10.8^{\mathrm{ab}}$ & $0.72 \pm 0.001^{\mathrm{ab}}$ & $46.60 \pm 0.48^{\mathrm{b}}$ & $7.51 \pm 0.07^{\mathrm{b}}$ \\
6 & $668 \pm 10.9^{\mathrm{ab}}$ & $0.72 \pm 0.001^{\mathrm{a}}$ & $46.50 \pm 0.49^{\mathrm{b}}$ & $7.43 \pm 0.08^{\mathrm{b}}$ \\
7 & $705 \pm 10.6^{\mathrm{b}}$ & $0.75 \pm 0.001^{\mathrm{c}}$ & $48.71 \pm 0.47^{\mathrm{c}}$ & $7.82 \pm 0.09 \mathrm{c}$ \\
8 & $684 \pm 11.5^{\mathrm{b}}$ & $0.74 \pm 0.001^{\mathrm{bc}}$ & $48.15 \pm 0.45^{\mathrm{c}}$ & $7.91 \pm 0.09 \mathrm{c}$ \\
\hline EEM & \pm 10.68 & \pm 0.001 & \pm 0.48 & \pm 0.09 \\
\hline
\end{tabular}

abcd Diferentes letras representan diferencia significativa $(P<0.05)$, excepto cenizas y fósforo en tibias $(P<0.01)$.

Para conocer el porcentaje de fósforo inorgánico liberado por las enzimas empleadas, se utilizaron las curvas estándar de porcentaje de cenizas y fósforo en tibias y se traspuso a la ecuación los valores obtenidos con las fitasas del siguiente modelo $\left(X=(\beta o-Y) / \beta_{1}\right)$. Donde: $X=$ porcentaje de $\mathrm{Pi}$ liberado por la enzima, $\beta o=$ ordenada al origen, $Y=$ ganancia de peso, eficiencia alimenticia, porcentaje de cenizas en tibias y porcentaje de fósforo en tibias y $\beta_{1}=$ coeficiente de regresión. También se realizó la comparación de medias con la prueba de Tukey considerando diferencia significativa cuando $P<0.05$.

\section{RESULTADOS}

Los resultados promedio de ganancia de peso, eficiencia alimenticia y porcentajes de cenizas y $\mathrm{P}$ en tibias en 14 días de experimentación, se observan el Cuadro 2. Se aprecia que la suplementación de fósforo a partir de FMD o la suplementación de las fitasas mejoraron $(P<0.05)$ las variables productivas al incrementarse el contenido de fósforo en las dietas y al incluir las fitasas. También se aprecia que los porcentajes de cenizas y fósforo en tibias con las dos fitasas bacterianas, liberaron cantidades similares $(P>0.05)$ de Pi tanto en la dosis 500 FTU como en la de 1,000 FTU. El contenido porcentual de cenizas y fósforo en tibias, se utilizó para calcular la liberación de fósforo obtenida con las fitasas en relación al fosfato monodicálcico.

Los resultados del porcentaje de cenizas en tibias mostraron una respuesta lineal a los diferentes niveles de fósforo a partir de FMD adicionados en la dieta $(P<0.05)$. El porcentaje de cenizas aumentó linealmente (cenizas, \% $=43.993+24.429 \times \% \mathrm{Pi}$ adicionado, $\mathrm{R}^{2}=0.34$ ) conforme el porcentaje de fósforo en la dieta se incrementó (Figura 1). Al trasponer en la ecuación el porcentaje de cenizas en tibias, la dosis de 500 FTU de las fitasas de Citrobacter braakii y Escherichia coli se obtuvo en promedio una liberación de $0.105 \%$. Sin embargo, para la inclusión de 1,000 FTU de ambas fuentes de fitasa, se encontró que la liberación de fósforo fue de $0.180 \%$.

Al analizar la cantidad de fósforo depositado en las tibias, se determinó que la dieta basal (sin Pi adicionado), obtuvo el porcentaje de fósforo más bajo ( $7.07 \%)$, el cual gradualmente se incrementó al aumentar la cantidad de Pi en la dieta ( $P$ en tibia, $\%=7.062+4.28 \times \% \mathrm{Pi}$ adicionado), y el tratamiento 4 obtuvo $8.0 \%$ de fósforo en tibia (Cuadro 2). De igual manera la adición de fitasa a la dieta basal incrementó el porcentaje de $\mathrm{P}$ en las tibias. 
Figura 1. Porcentajes de fósforo liberado en base al contenido de cenizas en tibias con 500 y $1,000 \mathrm{FTU} / \mathrm{kg}$ de fitasas de Citrobacter braakii o Escherichia coli

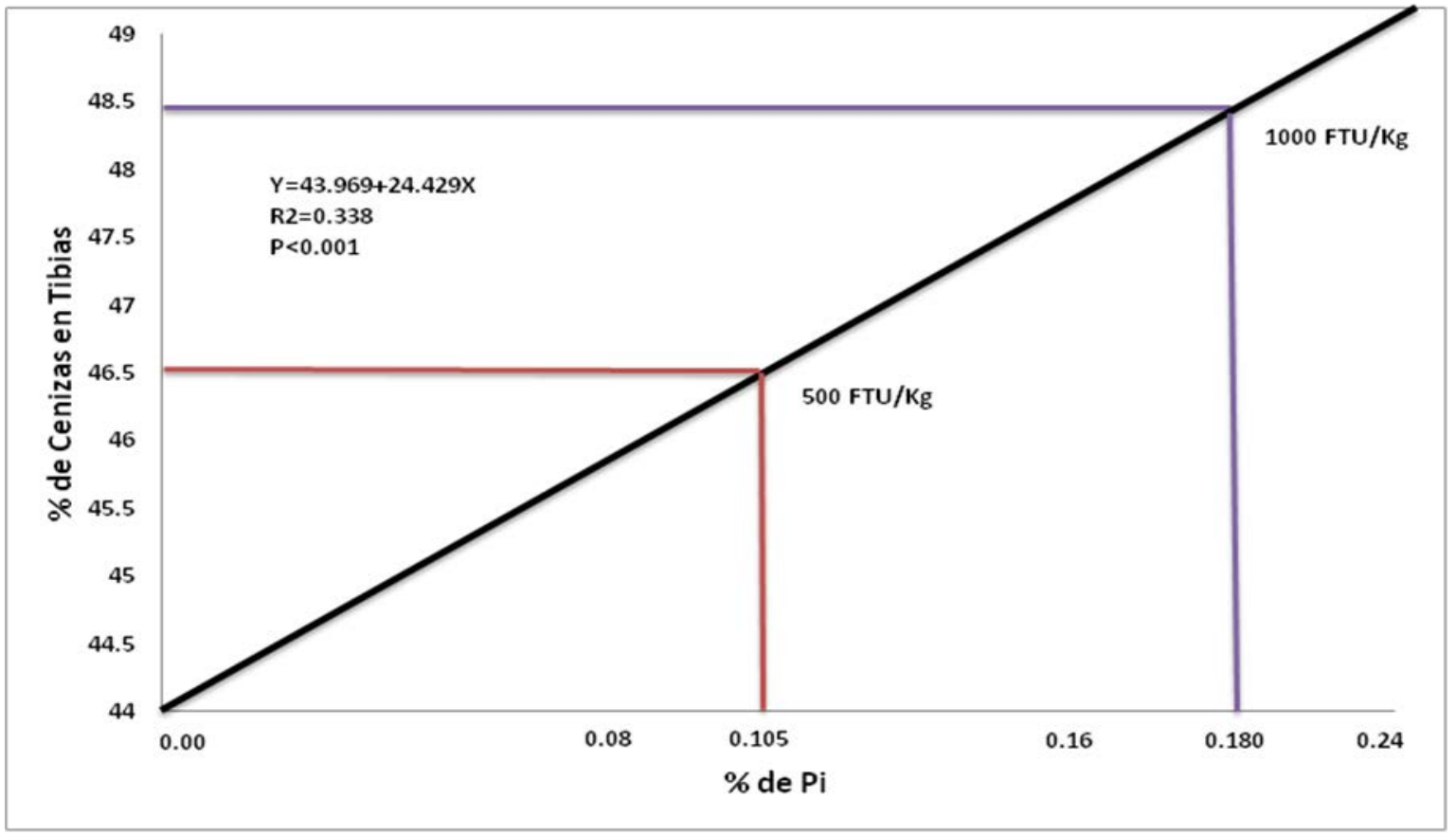

Al trasponer en la ecuación el porcentaje de Pi en tibias, se observó que al adicionar $500 \mathrm{FTU} / \mathrm{kg}$ de las dos fitasas (Escherichia coli y Citrobacter braakii) se tuvo una liberación de fósforo en promedio de $0.097 \%$. Por otro lado, al incrementar la dosis a $1,000 \mathrm{FTU} / \mathrm{kg}$ de ambas fuentes de fitasas, se aumentó la cantidad de fósforo liberado en un promedio de $0.185 \%$ (Figura 2).

Al promediar los valores de liberación de $\mathrm{P}$ en el contenido porcentual de cenizas y $P$ en las tibias, con las dos fitasas, la cantidad liberada fue de $0.102 \%$ con $500 \mathrm{FTU} / \mathrm{kg}$ y de $0.182 \%$ para $1,000 \mathrm{FTU} / \mathrm{kg}$ de alimento en dietas sorgo-soya.

\section{DISCUSIÓN}

El propósito principal de suplementar fitasas en dietas para aves, es mejorar el aprovechamiento del fósforo fítico y reducir la cantidad de fosfato en la dieta, ya que la fitasa hidroliza al fitato y libera el fósforo inorgánico, que es utilizado por el ave para cubrir el requerimiento de este mineral.
En este estudio la ganancia de peso se mejoró conforme se incrementó la cantidad de Pi en la dieta deficiente en este mineral; este efecto fue similar a lo encontrado por otros autores ${ }^{(15)}$, quienes al evaluar este parámetro con diferentes niveles de fósforo en la dieta (menores a $0.45 \%$ de Pi), las aves tuvieron un mayor consumo de alimento y por lo tanto de fósforo, lo que resultó en una mayor ganancia de peso al acercarse al requerimiento de este mineral. Driver et $a^{\left({ }^{16)}\right.}$ realizaron un estudio, utilizando dietas deficientes de fósforo (menores de $0.42 \%$ ) en pollos de 14 y 28 días de edad, y los parámetros productivos mejoraron cuando las aves tuvieron mayor consumo de fósforo en la dieta.

El nivel óptimo biológico de fósforo disponible en la dieta para máxima ganancia de peso en el período de 1 a 21 días de edad sugerido por Julian y Summers ${ }^{(17)}$ y Perney et $a{ }^{(18)}$, es de $0.46 \%$. Este resultado es similar al $0.45 \%$ sugerido por el $\mathrm{NRC}^{(19)}$, pero un $13.3 \%$ mayor del $\mathrm{P}$ utilizado como máximo nivel en este experimento. Otros autores ${ }^{(20)}$ recomiendan $0.5 \%$ como el óptimo, sin embargo emplearon roca fosfórica que quizá tuvo una menor 
Figura 2. Porcentajes de fósforo liberado en base al contenido de fósforo en tibias con 500 y 1,000 FTU/kg de fitasas de Citrobacter braakii o Escherichia coli

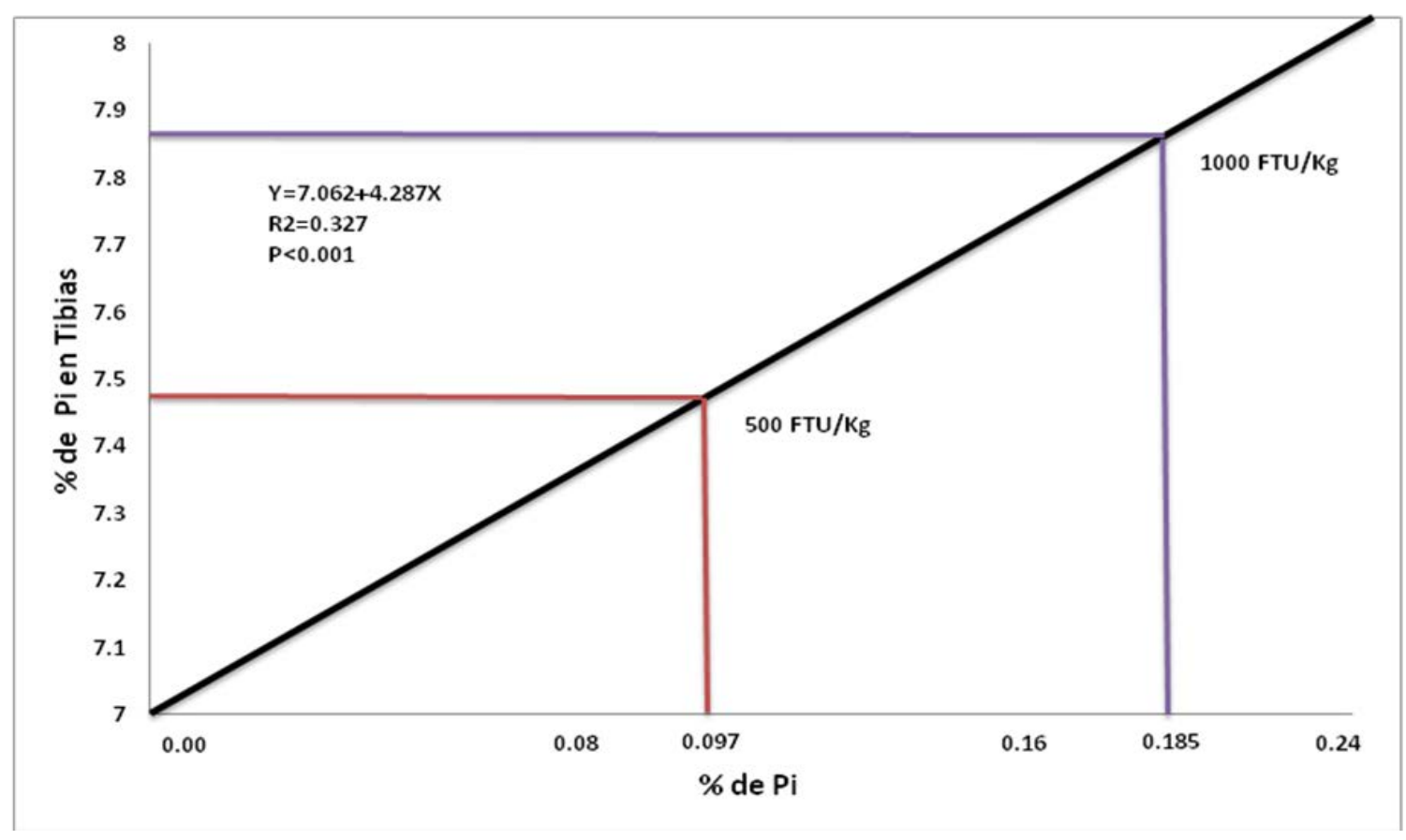

disponibilidad de fósforo. Los resultados de ganancia y eficiencia para los niveles máximos de $P$ suplementado en nuestro experimento son acordes a los esperados para el tipo de pollo utilizado cuando es alimentado sin restricción en $\mathrm{P}$ en la dieta.

Olukosi y Fru-Nji( ${ }^{(8)}$ evaluaron tres niveles de fitasa de Citrobacter braakii (0, 1,000 y 2,000 FTU) en pollos durante 14 días en dietas maíz-trigo-soya con la matriz de la fitasa en la dieta y otra dieta sin la matriz de la fitasa, con dos proporciones de Ca:P (2: 1 y $2.5: 1$ ). Los resultados indicaron que solo hubo efecto significativo en la utilización de fósforo, calcio y energía, al adicionar ambas dosis de fitasas en la dietas sin la matriz de la fitasa, y también con efecto benéfico en las dietas que contenían una relación de calcio:fósforo de 2:1. En un estudio similar ${ }^{(9)}$ reportan mejores resultados para ganancia de peso, eficiencia alimenticia; así como cenizas, fósforo, calcio, magnesio, potasio y zinc en tibias, al suplementar 1,000 y 2,000 FTU/kg de fitasa en la dieta, sin existir diferencia entre las dosis empleadas.
En un trabajo de meta-análisis con artículos publicados hasta el año 2013, donde se recopiló información de 286 artículos sobre la retención de fósforo en pollos de engorda, resumieron que la suplementación de fitasa (1,039 FTU/kg de dieta) para pollos de engorda, incrementó la retención de fósforo en $0.086 \%^{(21)}$.

Al reducir los niveles de fósforo inorgánicos en la dieta disminuye el porcentaje de cenizas en tibias, esto debido a que el $80 \%$ del fósforo total del organismo es depositado en tejido óseo(22).

Los resultados mostraron que la adición de las fitasas microbianas procedentes de Citrobacter braakii o Escherichia coli mejoraron de manera similar la biodisponibilidad del fósforo proveniente del fósforo fítico de la pasta de soya y el sorgo; resultados similares reportan que el empleo de 500 FTU/kg fue eficaz en mejorar el crecimiento, cenizas en tibias y retención de fósforo en pollos de engorda, con la liberación de $0.08 \%$ de fósforo disponible ${ }^{(23)}$. Augspurger et $a^{(10)}$ al evaluar $500 \mathrm{FTU} / \mathrm{kg}$ de fitasa de $E$. coli en dietas para pollos mencionan liberaciones de $0.125 \%$ de fósforo. 
En el presente estudio se encontró que ambas fitasas liberaron cantidades similares de fósforo, y se ha demostrado que la fitasa cuando se utiliza en dosis elevadas mejora el rendimiento productivo(24). Sin embargo, Cortes et a/4) encontraron mayor ganancia de peso, conversión alimenticia y energía metabolizable aparente en pollos de 1 a 19 días de edad con el empleo de fitasa proveniente de Peniophora licii, además de permitir reducir en la dieta (sorgo-soya) $0.1 \%$ de fósforo sin afectar el rendimiento productivo.

También se menciona(11) que al evaluar una dosis de 500 FTU de una fitasa microbiana en dietas con $0.22 \%$ de $\mathrm{Pi}$, obtuvieron un incremento en la ganancia de peso, y la mineralización en tibias; al aumentar la dosis a 1,000 FTU la cantidad de fósforo que liberó la enzima aumentó en 0.07,\%, obteniéndose resultados muy similares a los encontrados en el presente estudio. Los mismos autores utilizaron una tercera dosis de fitasa microbiana de 2,000 FTU con la misma cantidad de fósforo en la dieta $(0.22 \%)$ sin obtener diferencias significativas entre esta dosis y la utilización de 1,000 FTU.

En este estudio se incrementó $78.4 \%$ más la liberación de fósforo a partir del fitato, al aumentar la dosis de 500 FTU a 1,000 FTU para ambas fitasas provenientes de Citrobacter braakii y Escherichia coli. Para lograr una máxima hidrólisis del fósforo fítico, los niveles de dosificación y el contenido de fitato de la dieta, van a tener un papel importante en la respuesta productiva ${ }^{(25)}$.

\section{CONCLUSIONES E IMPLICACIONES}

Con los resultados obtenidos en el presente estudio, se puede concluir que el empleo de ambas fitasas de Citrobacter braakii o Escherichia coli a la dosis de $500 \mathrm{FTU} / \mathrm{kg}$ liberaron $0.102 \%$ de fósforo y con la inclusión de 1,000 FTU/kg (megadosis) liberaron $0.182 \%$, lo equivalente a $78.4 \%$ más de aprovechamiento del fósforo fítico en dietas sorgosoya para pollos en crecimiento.

\section{LITERATURA CITADA}

1. Li W, Angel R, Kim SW, Brady K, Yu S, Plumstead PW. Impacts of dietary calcium, phytate and nonphytate phosphorus concentrations in the presence or absence of phytase on inositol hexakisphosphate (IP6) degradation in different segments of broilers digestive tract. Poult Sci 2016;95:1-9.

2. Adeola O, Cowieson AJ. Opportunities and challenges in using exogenous enzymes to improve non-ruminant animal production. J Anim Sci 2011;89:3189-3218.

3. Ravindran V. Feed enzymes: The Science, practice, and metabolic realities. J Appl Poult Res 2013;22:628-636.

4. Cortes CA, Fuente MB, Fernández TS, Mojica EMC, Avila GE. Evaluación de la presencia de una fitasa microbiana (Peniophora lycii) en dietas sorgo-soya deficientes en fósforo para pollos de engorda, sobre la digestibilidad ileal de proteína, aminoácidos y energía metabolizable. Vet Mex 2007;38:21-29.

5. Cowieson AJ, Acamovik T, Bedford MR. Phytic acid and phytase: Implications for protein utilization by Poultry. Poult Sci 2006; 85:878-885.

6. Selle PH. Microbial phytase in poultry nutrition, Anim Feed Sci Technol 2007; 135: 1-41.

7. Cowieson AJ, Bedford MR. The effects of phytase and phytic acid on the loss of endogenous amino acids and minerals from broiler chicken. Poult Sci 2004; 93:3021-3031.

8. Olukosi OA, Fru-Nji F. The interplay of dietary nutrient and varying calcium to phosphorus rations on efficacy of a bacterial phytase: 2. Il eal and total tract nutrient utilization. Poult Sci 2014;93:30443052.

9. Olukosi OA, Fru-Nji F. The interplay of dietary nutrient and varying calcium to total phosphorus ration on efficacy of a bacterial phytase: 1 . Grow performance and tibia mineralization. Poult Sci 2014; 93:3037-3043.

10. Auguspurger NR, Webel DM. Efficacy of an E. coli phytase expressed in yeast for releasing phytate-bound phosphorus in young chicks and pigs. J Anim Sci 2003; 81:474-483.

11. Bedford, MR, Partridge GG. Enzymes in farm animal nutrition. $2^{\text {nd }}$ ed. CABI Publishing, UK, 2010.

12. Walk CL, Santos TT, Bedford MR. Influence of superdoses of a novel microbial phytase on growth performance, tibia ash, and gizzard phytate and inositol in young broilers. Poult Sci 2014; 93:1172-1177.

13. Association of Official Analytical Chemists. Official Methods of Analysis of AOAC International. $18^{\text {th }}$ ed. AOAC Int., Gaithersburg, MD., 2006.

14. Statistical Package for the Social Sciences Inc. SPSS for Windows Version 17.0. 2014.

15. Tamim NM, Angel R, Christman M. Influence of dietary calcium and phytase on phytate phosphorus hydrolysis in broiler chickens. Poult sci 2004; 83:1358-1362.

16. Driver J, Atencio A, Edwuards H, Pesti G. Improvements in nitrogen corrected aparent metabolizable energy of peanut meal in response to phytase suplementation. Poult Sci 2006;85:96-93.

17. Julian JR, Summers J, Wilson JB. Right ventricular failure and ascites in broiler chickens caused phosphorus-deficient diets. Avian Dis 1985; 30:453-459. 
18. Perney KM, Cantor AH, Straw ML, Herkelman KL. The effect of dietary phytase on growth and phosphorus utilization of broiler chick. Poult Sci 1993; 72:2106-2114.

19. NRC. Nutrient requirements of poultry. 9th revised ed. National Research Council, National Academy Press, Washington DC. 1994.

20. Ardon GA, Barillas A. Efecto del nivel de fósforo en la ganancia de peso de pollos de engorda [tesis licenciatura]. México: Universidad Autónoma de Chapingo; 1995.

21. Bougouin A, Appuhammy J ADRN, Kebreab E, Dijkstra J, Kwakkel RP, France J. Effects of phytase supplementation on phosphorus retention in broilers and layers: A meta-analysis. Poult Sci 2014; 93: 1981-1992.
22. Zeller E, Schollenberger M, Kuhn I, Rodehutscord M. Hydrolysis of phytate and formation of inositol phosphate isomers without or with supplemented phytases in different segments of the digestive tract of broilers. J Nutr Sci 2015;4:1-12.

23. Onyango EM, Bedford MR, Adeola O. Efficacy of an evolved Escherichia coli phytase in diets of broiler chicks. Poult Sci 2005; 84:248-255.

24. Shirley RB, Edward HM Jr. Graded levels of phytase past industry standards improves broiler performance. Poult Sci 2003;82:671680.

25. Adeola O, Cowieson AJ. Opportunities and challenges in using exogenous enzymes to improve nonruminant animal production. J Anim Sci 2011;89:3189-3218. 